\title{
Use of DOTATATE PET/CT Scan in the Diagnosis and Staging of Thymic Atypical Carcinoid Tumor in a Patient with Secondary ACTH-dependent Cushing Syndrome: Look Twice and Cut Once
}

\author{
1 John Agzarian, ${ }^{2}$ Hisham Quandeel, ${ }^{3}$ Irina Bancos, ${ }^{4}$ Geoffrey B Johnson, ${ }^{5}$ Stephen C Scharf, ${ }^{6}$ Geoffrey B Thompson \\ ${ }^{7}$ Joanne Yi, ${ }^{8}$ Xiaotun Zhang, ${ }^{9} \mathrm{~K}$ Robert Shen
}

\begin{abstract}
Neuroendocrine thymic tumors represent the least common type of primary thymic tumor with a prevalence of 2 to $5 \%$. We present a case of locally advanced thymic atypical carcinoid tumor diagnosed incidentally while investigating progressive Cushing syndrome. Computed tomography (CT) scan demonstrated a large $2.9 \mathrm{~cm}$ exophytic thyroid nodule and a $2.0 \mathrm{~cm}$ anterior mediastinal mass. Biopsy of the thyroid nodule demonstrated benign thyroid tissue, and octreotide scan revealed avid uptake in the right thyroid lobe with minimal uptake in the thymic tumor. ${ }^{68}$ Gallium-1,4,7,10-tetraazacyclododecane-N,N',N"N'"tetraacetic acid-D-Phe ${ }^{1}, \mathrm{Tyr}^{3}$-octreotate (Ga-68 DOTATATE) positron emission tomography (PET)/CT scan showed intense uptake in the thyroid gland followed by a moderate amount of activity in the anterior mediastinal mass. The patient underwent a median sternotomy and radical thymectomy with en bloc resection of the left innominate vein and primary repair of the superior vena cava (SVC). On further evaluation, the presumed thyroid nodule appeared contiguous with the thymic lesion and separate from the thyroid. Final pathology demonstrated a poorly differentiated $4.4 \mathrm{~cm}$ atypical carcinoid tumor with nine positive lymph nodes. On the third postoperative day, the serum cortisol level precipitously decreased to a level of $6.8 \mu \mathrm{g} / \mathrm{dL}$.

This case highlights the challenges surrounding the diagnosis of hormonally active thymic carcinoids and demonstrates the utility of Ga-68 DOTATATE PET scan in localizing somatostatinpositive neuroendocrine tumors and surgical planning.
\end{abstract}

\footnotetext{
${ }^{1,9}$ Thoracic Surgeon, ${ }^{2}$ Cardiac Surgeon, ${ }^{3}$ Endocrinologist ${ }^{4,5}$ Radiologist, ${ }^{6}$ Endocrine Surgeon, ${ }^{7,8}$ Pathologist

1,2,9 Department of Thoracic Surgery, Mayo Clinic, Rochester Minnesota, USA

${ }^{3}$ Department of Endocrinology, Mayo Clinic, Rochester Minnesota, USA

${ }^{4}$ Department of Radiology and Immunology, Mayo Clinic Rochester, Minnesota, USA

${ }^{5}$ Department of Radiology, Lennox Hill Hospital, New York, USA

${ }^{6}$ Department of Endocrine Surgery, Mayo Clinic, Rochester Minnesota, USA

${ }^{7,8}$ Department of Anatomic Pathology, Mayo Clinic, Rochester Minnesota, USA

Corresponding Author: John Agzarian, Thoracic Surgeon Department of Thoracic Surgery, Mayo Clinic, Rochester Minnesota, USA, e-mail: john.agzarian@medportal.ca
}

Keywords: DOTATATE, Mediastinal, Positron emission tomography, Thymic carcinoid.

How to cite this article: Agzarian J, Quandeel H, Bancos I, Johnson GB, Scharf SC, Thompson GB, Yi J, Zhang X, Shen KR. Use of DOTATATE PET/CT Scan in the Diagnosis and Staging of Thymic Atypical Carcinoid Tumor in a Patient with Secondary ACTH-dependent Cushing Syndrome: Look Twice and Cut Once. World J Endoc Surg 2018;10(2):127-133.

\section{Source of support: Nil}

Conflict of interest: None

\section{INTRODUCTION}

The thymus is a primary source of neuroendocrine tumors in $0.4 \%$ of all carcinoid tumors, corresponding to an annual incidence in the United States of 0.2 per million. ${ }^{1,2}$ Neuroendocrine thymic tumors represent the least common type of primary thymic tumor with a prevalence of 2 to $5 \%{ }^{3,4}$ Neuroendocrine tumors arising in the thymus are classified as low-grade (typical carcinoid), intermediate (atypical carcinoid), and high grade (large cell neuroendocrine carcinoma and small cell carcinoma). Generally, thymic carcinoids tend to be aggressive neoplasms. There is a high risk of local invasion, and mediastinal lymph node metastases occur in nearly $50 \%$ of patients. ${ }^{5,6}$ Approximately, 20 to $40 \%$ of patients have distant metastases at the time of diagnosis, with the most common sites of distant spread being the lung, pleura, bone, liver, pancreas, and chest wall. ${ }^{4}$

Paraneoplastic syndromes can develop in up to one-half of cases of thymic neuroendocrine tumors. Carcinoid syndrome, acromegaly, and hyponatremia secondary to syndrome of inappropriate antidiuretic hormone are uncommon endocrinopathies. ${ }^{7-9}$ The most common presentation (up to $50 \%$ ) is Cushing syndrome due to ectopic tumor production of adrenocorticotropic hormone (ACTH) ${ }^{10,11}$ Paraneoplastic syndromes are much more common in sporadic cases as compared with thymic carcinoids associated with multiple endocrine neoplasia type I-representing roughly $25 \%$ of total cases. $^{12}$ 
We present a case of locally advanced thymic atypical carcinoid tumor diagnosed incidentally while investigating for progressive Cushing syndrome. The case highlights the challenges surrounding the diagnosis of hormonally active thymic carcinoids and demonstrates the utility of Ga-68 DOTATATE PET scanning. The Ga-68 DOTATATE is a novel radioactive imaging agent recently approved by the US Food and Drug Administration (FDA) and the Centers for Medicare and Medicaid Services (CMS) for use in PET imaging to help localize somatostatin-positive neuroendocrine tumors.

\section{CASE REPORT}

A 53-year-old nonsmoker, nondiabetic, and otherwise healthy female was referred to the endocrinology service for evaluation of Cushing syndrome initially identified after presenting to her primary care physician with a 2 months history of bilateral lower limb edema and refractory new-onset hypertension. Her previous surgical history includes a partial nephrectomy for recurrent urinary tract infections, strabismus correction, and in vitro fertilization. At her initial evaluation, her constellation of symptoms included facial swelling, weight gain (10-15 lb), insatiable appetite, hirsutism, easy bruising, insomnia, and muscle cramping of the hands and feet. Biochemical testing demonstrated evidence of hypokalemia, with a morning cortisol level of $44 \mu \mathrm{g} / \mathrm{dL}$ (normal range 7-25 $\mathrm{\mu g} / \mathrm{dL}$ ) and an ACTH of $240 \mathrm{pg} / \mathrm{mL}$ (normal range 10-60 $\mathrm{pg} / \mathrm{mL}$ ). Renin plasma activity and aldosterone levels were within normal ranges ( 6 and $<4 \mathrm{ng} / \mathrm{dL}$ respectively). Cortisol in 24-hour urine collection was elevated at $715 \mu \mathrm{g}$ (normal ranges 3.5-45 $\mu$ ), and cortisol after $1 \mathrm{mg}$ dexamethasone administration was not suppressed at $22 \mu \mathrm{g} /$ $\mathrm{dL}$ (normal $<1.8 \mu \mathrm{g} / \mathrm{dL}$ ), confirming Cushing syndrome. Brain magnetic resonance imaging (MRI) was performed to assess for the presence of a pituitary tumor. Inferior petrosal sinus sampling (IPSS) revealed no difference between the peripheral and petrosal sinus ACTH levels.

As IPSS suggested an ectopic source of ACTH production, further work-up was conducted in search for a neuroendocrine tumor. Computed tomography scan of the abdomen and pelvis demonstrated mild nodularity of the adrenals bilaterally with no definitive adenomas and without other abnormalities. Computed tomography scan of the chest depicted scattered bilateral indeterminate pulmonary nodules $(<3 \mathrm{~mm})$, a large exophytic thyroid nodule $(2.9 \times 1.9 \mathrm{~cm})$ extending from the inferior right lobe into the superior mediastinum, and an anterior mediastinal mass $(2.0 \times 1.1 \mathrm{~cm})$ at the confluence of the brachiocephalic vein into the middle mediastinum. Magnetic resonance imaging of the chest further characterized the anterior mediastinal mass between the right brachiocephalic vein and innominate artery, which was suspicious for thymic neoplasm, thymoma, or carcinoma. Ultrasound-guided biopsy of the thyroid nodule yielded benign thyroid tissue, and her calcitonin level was normal $(<5 \mathrm{pg} / \mathrm{dL})$ effectively ruling out medullary thyroid cancer as a source of her ACTH. A single-photon emission CT octreotide scan was performed, surprisingly revealing avid uptake in the right thyroid lobe with only minimal uptake in the thymic tumor.

Prior to the octreotide scan, the most likely source of ectopic hormone appeared to be the thymic tumor. However, the octreotide scan results conflicted that conjecture. The presumptive treatment plan at that time was for thymectomy and if the cortisol levels did not decrease postoperatively, consideration of hemithyroidectomy would be given. Bilateral adrenalectomy was considered the final option if removal of the thymus and thyroid nodule did not obliterate the ectopic ACTH production. The patient was accordingly referred to thoracic surgery. After further deliberation, the patient was referred for a Ga-68 DOTATATE PET-CT scan to more definitively localize the possible ACTH source. This scan showed most intense activity in the thyroid gland followed by a moderate amount of activity in the anterior mediastinal mass (Figs 1 and 2).

In light of the clinical findings, the decision was made to resect the anterior mediastinal mass, given that this represented the most likely source of ectopic ACTH. The patient underwent a median sternotomy and radical thymectomy with en bloc resection of the left innominate
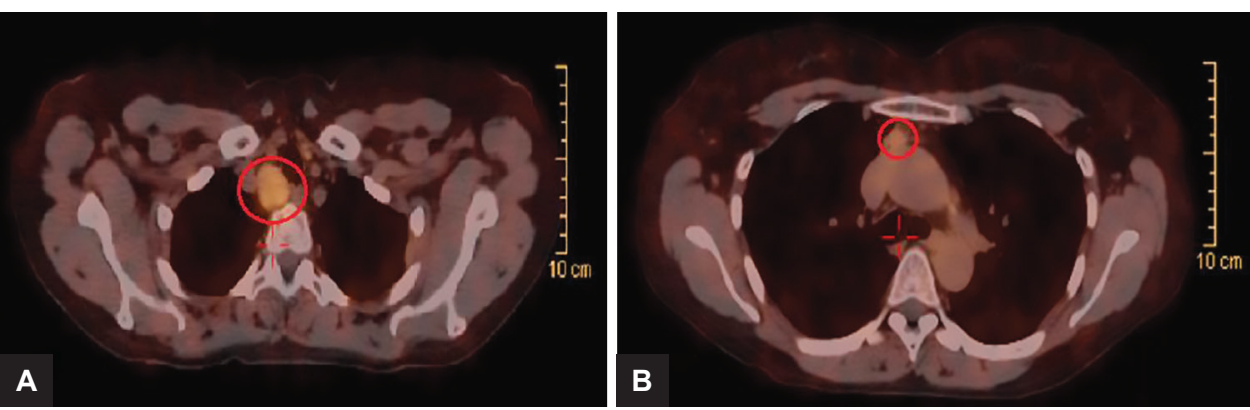

Figs 1A and B: Axial DOTA scan views of thymic tumor in superior mediastinum at confluence of SVC and innominate vein (A) and anterior mediastinum near aortic arch (B) 


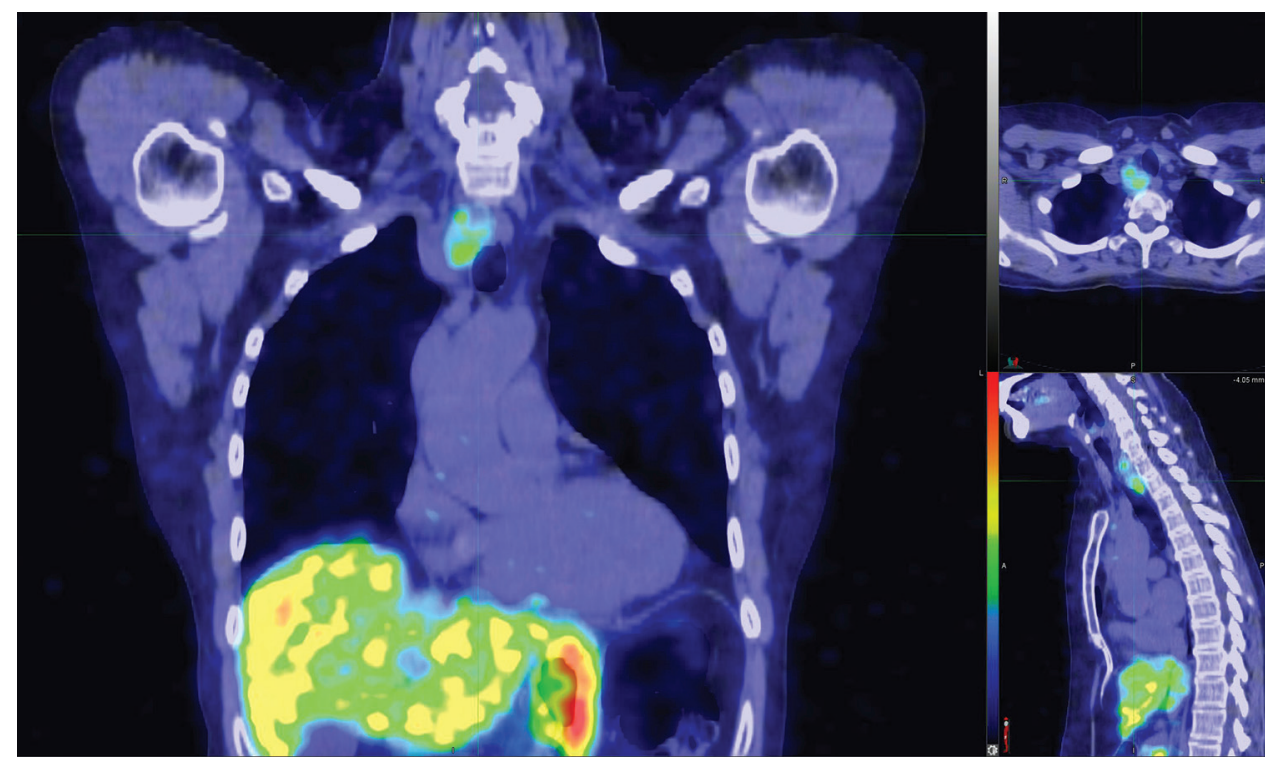

Fig. 2: DOTA scan images of avid tumor at superior mediastinal paratracheal region

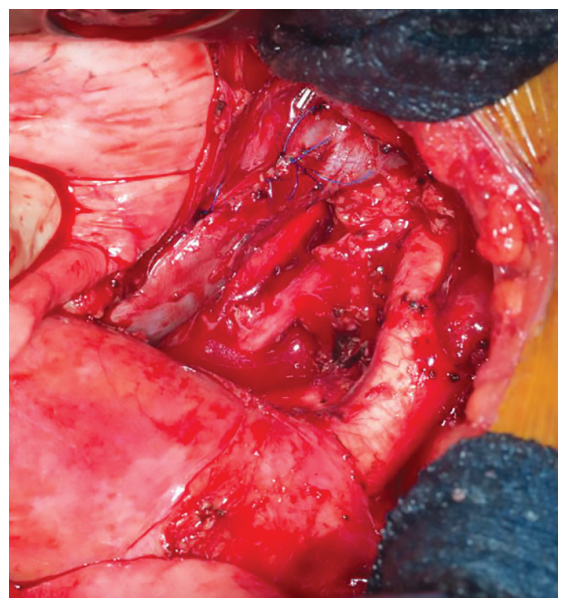

Fig. 3: Post-resection and primary repair of SVC

vein and primary repair of the SVC. The primary thymic mass was adherent to the pericardium at the level of the ascending aortic arch and required a resection of the anterior pericardium. On further evaluation, the presumed thyroid nodule appeared separate from the thyroid. Based on intraoperative inspection and frozen section pathologic analysis, this tissue was determined to be a conglomeration of metastatically involved lymph nodes. At this level, the tumor appeared densely adherent to the innominate vein at the SVC confluence. In order to achieve negative surgical margins, the tumor was resected en bloc with a portion of the SVC medial side wall (Figs 2 to 4). Frozen section analysis yielded negative resection margins. Final pathology demonstrated a poorly differentiated atypical carcinoid tumor forming multiple nodules in the anterior mediastinum (Fig. 5). The dominant nodule size was $4.4 \times 3.1 \times 1.5 \mathrm{~cm}$, with invasion of the wall of the left innominate vein. Multiple (9 out of 9) lymph nodes were positive for metastatic atypical

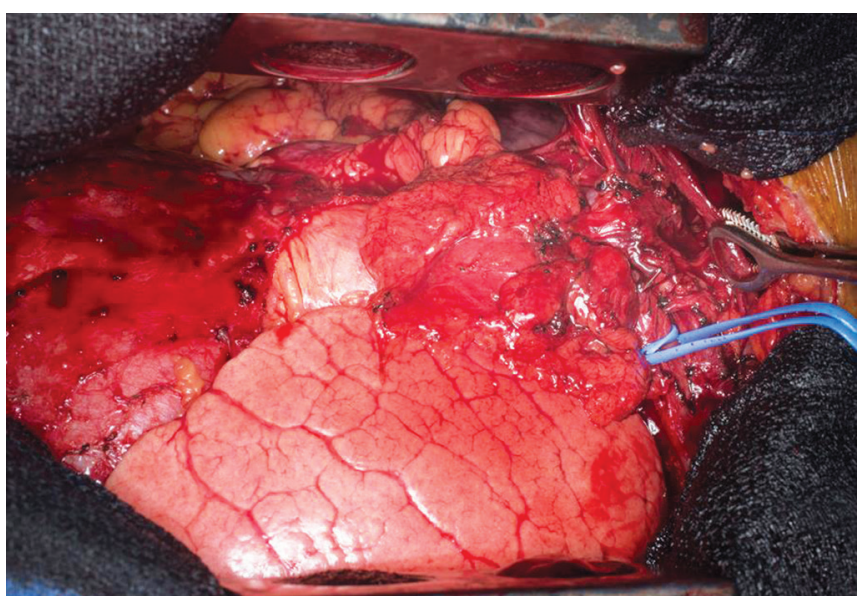

Fig. 4: Tumor involving confluence of left innominate vein and SVC

carcinoid - the largest of which was located within the superior horn of the thymus. The tumor cells were positive for cell adhesion molecule 5.2, chromogranin, trefoil factor 1, and ACTH. The Ki-67 index was low at approximately $5 \%$, and the mitotic count was $2 / 10$ high-power field(Fig. 6). Postoperatively, the patient's cortisol levels dropped appropriately. On the third postoperative day, the serum cortisol level precipitously decreased to a level of $6.8 \mathrm{\mu g} / \mathrm{dL}$. She was carefully monitored for symptoms of adrenal insufficiency, and she was started on a tapering dose of oral prednisone (Table 1 and Graph 1).

On follow-up, the patient was seen by medical and radiation oncology. While adjuvant chemotherapy was not recommended, she received postoperative external beam radiation to the surgical bed in the form of $50 \mathrm{~Gy}$ in 25 fractions. After several weeks, the patient began to experience an improvement in her symptoms. Her energy levels improved and her mood became less labile, with improved sleep, reduction in facial puffiness, and weight. 


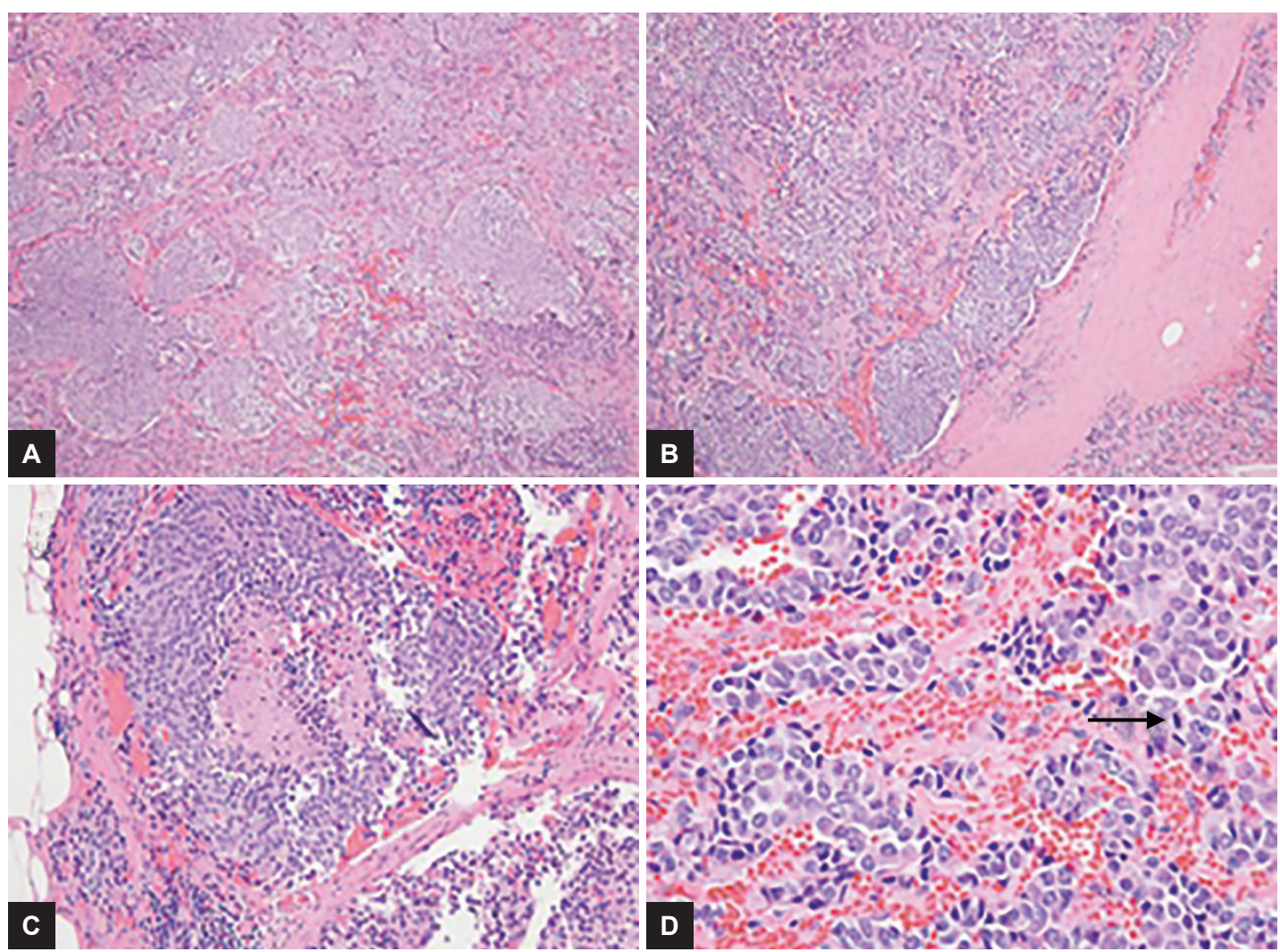

Figs 5A to D: Histological features of thymic atypical carcinoid tumor. (A) H\&E section shows neoplastic cells arranged in organoid nests, separated by intersecting bands of collagen. (B) Lymphovascular invasion and (C) punctate necrosis are present. (D) Neoplastic cells exhibit mild atypia with finely, granular chromatin (so called "salt and pepper pattern") and inconspicuous nucleoli. Arrow shows a mitotic figure
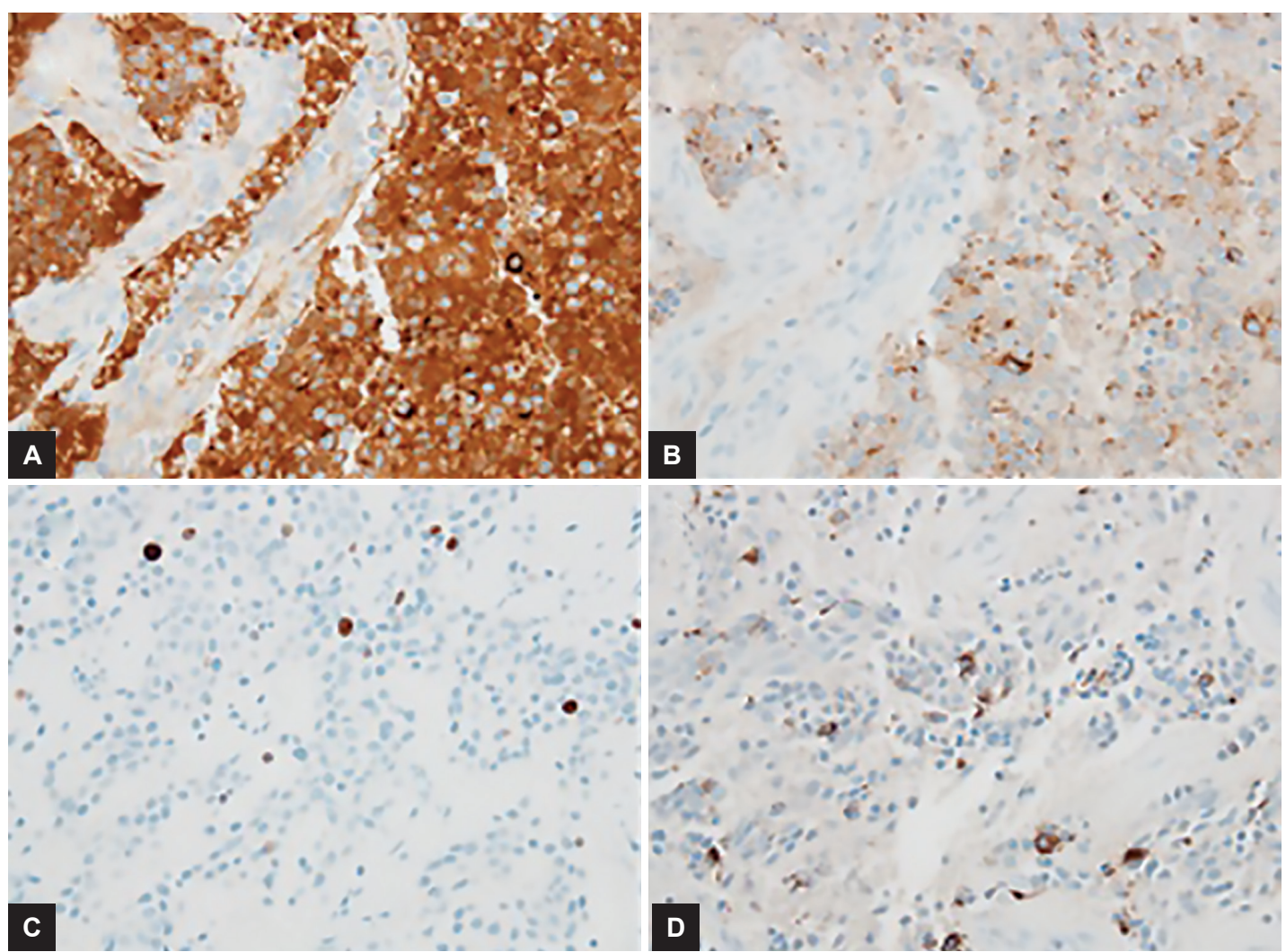

Figs 6A to D: Immunohistochemical stains of thymic atypical carcinoid tumor. (A) CAM5.2 (B) Chromogranin A (C) Ki67 (D) Adrenocorticotropic hormone 
Table 1: Pre- and postoperative trend in cortisol and values

\begin{tabular}{llll}
\hline Parameter & Date & Post-op-day \# & Value \\
\hline AM cortisol & July 29 & 1 & $31 \mathrm{mcg} / \mathrm{dL}$ \\
& July 30 & 2 & $19 \mathrm{mcg} / \mathrm{dL}$ \\
& July 31 & 3 & $6.8 \mathrm{mcg} / \mathrm{dL}$ \\
& December 5 & 127 & $7.4 \mathrm{mcg} / \mathrm{dL}$ \\
ACTH & July 28 & 0 & $64 \mathrm{pg} / \mathrm{mL}$ \\
& July 29 (00:31) & 1 & $32 \mathrm{pg} / \mathrm{mL}$ \\
& July 29 (09:05) & 1 & $31 \mathrm{pg} / \mathrm{mL}$ \\
& July 29 (14:32) & 1 & $48 \mathrm{pg} / \mathrm{mL}$ \\
& July 29 (23:20) & 1 & $17 \mathrm{pg} / \mathrm{mL}$ \\
& July 30 & 2 & $19 \mathrm{pg} / \mathrm{mL}$ \\
& July 31 & 3 & $17 \mathrm{pg} / \mathrm{mL}$ \\
& September 2 & 33 & $20 \mathrm{pg} / \mathrm{mL}$ \\
& December 5 & 127 & $20 \mathrm{pg} / \mathrm{mL}$ \\
\hline
\end{tabular}

Operative date: July 28, 2016

\section{DISCUSSION}

Given the aggressive nature of thymic carcinoids and the high rate of local recurrence, diagnostic confirmation is important in assessing tumor resectability-with complete $\mathrm{R} 0$ resection being the mainstay of treatment. ${ }^{13}$ The preoperative assessment of this patient led to the presumption of a small focal thymic mass as the potential source of ectopic ACTH. This was challenged by the positive octreotide and Ga-68 DOTATATE uptake of what was misinterpreted as a retrosternal thyroid nodule. Operative confirmation clarified that the mass was contiguous with the focal abnormality at the confluence of the innominate vein and SVC. This better fits with the classic presentation of thymic neuroendocrine tumors as locally invasive anterior mediastinal masses. Computed tomography and MRI findings did not conclusively depict the contiguous nature of these two lesions, and nuclear medicine imaging (specifically DOTATATE PET scan) was ultimately accurate in identifying the thymic mass as the source of ectopic ACTH in this patient.

On CT scan, these tumors typically present as large, lobulated invasive anterior mediastinal masses with heterogeneous enhancement and possible areas of hemorrhage and necrosis. There are, however, no pathognomonic radiographic features that distinguish thymic carcinoids from other thymic malignancies or nonthymic lesions. Moreover, fluorine 18-fluorodeoxyglucose (FDG) PET scans have limited role in diagnosis and staging of neuroendocrine tumors, given their often low glucose metabolic rate and low FDG activity. ${ }^{14}$ Some evidence, however, supports the utility of FDG PET scans for intermediate and high-grade neuroendocrine tumor. ${ }^{15,16}$ The overexpression of somatostatin receptors allows for the use of radiolabeled somatostatin analogs in identifying these tumors. In addition, radiolabeled nuclear imaging can play a role in excluding a distant primary

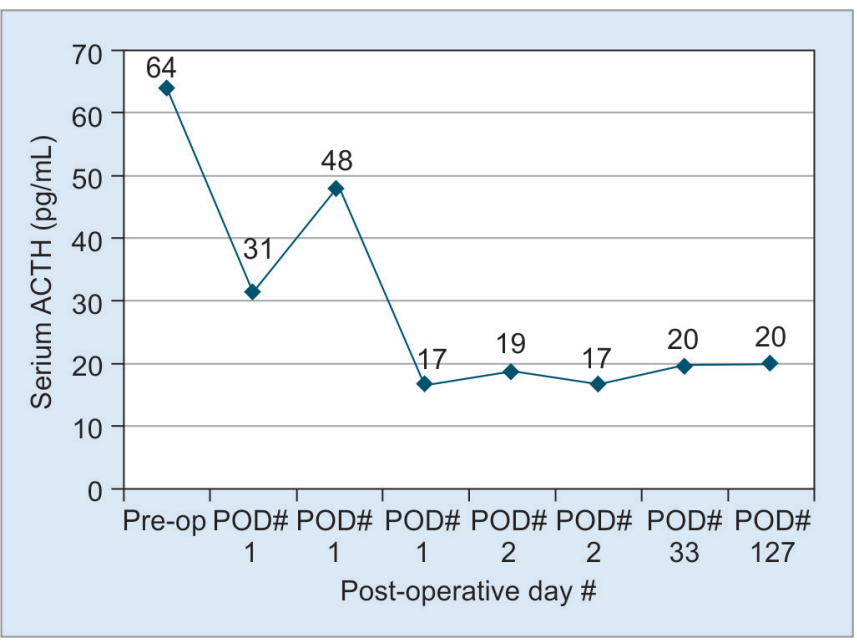

Graph 1: Trends in serum ACTH following resection

site of disease with metastases to the thymus (despite the rarity of this phenomenon), and in identifying metastatic disease from a primary thymic source. ${ }^{17,18}$ Radiolabeled nucleotide uptake can also determine if a patient would potentially benefit from somatostatin analog therapy, or the currently experimenta ${ }^{177}$ Lu-DOTATATE therapy. ${ }^{19,20}$

To date, the majority of evidence regarding the use of Ga-68 DOTATE PET is from the pulmonary and midgut carcinoid literature. The Ga-68 DOTATATE is a selective somatostatin receptor II ligand that can be used to radiolabel and therefore to detect neuroendocrine tumors with PET scanner.

Kayani et $\mathrm{al}^{21}$ evaluated the performance of DOTATATE in the detection of pulmonary neuroendocrine tumors. The results demonstrated that DOTATATE was superior to FDG in detecting tumors of typical histology and was better able to delineate endobronchial tumors from adjacent atelectasis. The DOTATATE uptake was however less in atypical carcinoids and higher grade neuroendocrine lesions (median standardized uptake value $_{\max }$ of 33 vs 2.8), while FDG PET was conversely higher in high-grade lesions. This can be explained by the fact that the more poorly differentiated carcinoid tumors are more likely to lose somatostatin receptor expression. ${ }^{22}$ These findings were later echoed by Lococo et $\mathrm{al}^{23}$ in a multicenter comparison of FDG vs DOTATATE PET/CT scan in pulmonary carcinoid. The DOTA PET/CT had a better detection rate as compared with FDG PET/CT (79 vs $55 \%$ respectively), with higher uptake values for DOTA scans. Fluorodeoxyglucose PET/CT was, however, superior in the detection of atypical carcinoid. These studies support a combined approach to diagnosing and staging pulmonary neuroendocrine tumors, where DOTATATE PET/CT can be used to distinguish typical carcinoid and low-grade lesions, while FDG PET/CT identifies atypical malignancies. 
There is a paucity of research delineating the role and utility of DOTA PET/CT scans in the setting of thymic neuroendocrine tumors. In this case presentation, the patient was not investigated with FDG PET/CT, and therefore comparison between both modalities is not possible. In a comparative systematic review of conventional and nuclear medicine imaging in 231 patients with ectopic ACTH syndrome, DOTATATE PET/CT demonstrated the highest sensitivity in the setting of previously localized tumors as compared with octreotide scans and FDG PET (81.8 vs 48.95 and 51.7\% respectively). However, DOTATATE scans held the lowest sensitivity in localizing primary thymic sources of ectopic ACTH in comparison with octreotide scans and FDG PET (50 vs 85.7 and $62.5 \%$ respectively). ${ }^{24}$ Of 231 patients, only 23 were investigated with DOTATATE PET/CT. In a comparative analysis of the available imaging modalities to localize ectopic ACTH tumors, the authors recommended the use of DOTATATE PET/CT in the setting of negative MRI, or when octreotide scan and FDG PET/CT are either negative or nondiagnostic. ${ }^{24}$ This approach may be in part based on the fact that DOTATATE PET/CT was not FDA/CMS approved at the time.

As such, it appears as though the role of DOTATATE $\mathrm{PET} / \mathrm{CT}$ is most defined in confirming the hormonal activity of a previously localized tumor. In such a scenario, DOTATATE scan offers the highest sensitivity. In this specific case, following CT and MRI localization, this patient was further investigated with both octreotide scan and DOTATATE PET/CT-the two yielding concordant findings. The superior extent of the primary thymic lesion confounded the results of localizing studies, given the false assumption of thyroid origin. In addition, we did not use FDG/PET, which could have potentially proven useful given the atypical histology and aggressive nature of the tumor.

\section{CONCLUSION}

Taken together, this case presentation highlights the importance of an integrated approach in the work-up for thymic carcinoid tumors. The DOTATATE PET/CT is an emerging diagnostic modality that remains mostly unavailable in most centers in the United States, but whose availability is expected to expand dramatically in the near future. On June 1, 2016, the FDA approved Netspot, the first kit for the preparation of Ga-68 DOTATATE injection for PET imaging. The FDA granted priority review and orphan drug designation for Ga-68 DOTATATE. Its utility and role continue to be defined, particularly in the setting of occult thymic tumors. Extrapolating from available evidence, DOTATATE PET/CT can be used as an adjunct to other imaging techniques to identify hormonally active tumors and plan appropriate therapy. Future research is needed to differentiate the role of DOTATATE scan in comparison to other nuclear imaging modalities, such as octreotide scans.

\section{REFERENCES}

1. Gaur P, Leary C, Yao JC. Thymic neuroendocrine tumors: a SEER database analysis of 160 patients. Ann Surg 2010 Jun;251(6):1117-1121

2. Yao JC, Hassan M, Phan A, Dagohoy C, Leary C, Mares JE, Abdalla EK, Fleming JB, Vauthey JN, Rashid A, et al. One hundred years after "carcinoid": epidemiology of and prognostic factors for neuroendocrine tumors in 35,825 cases in the United States. J Clin Oncol 2008 Jun;26(18):3063-3072.

3. Goto K, Kodama T, Matsuno Y, Yokose T, Asamura H, Kamiya N, Shimosato Y. Clinicopathologic and DNA cytometric analysis of carcinoid tumors of the thymus. Mod Pathol 2001 Oct;14(10):985-994.

4. Chaer R, Massad MG, Evans A, Snow NJ, Geha AS. Primary neuroendocrine tumors of the thymus. Ann Thorac Surg 2002 Nov;74(5):1733-1740.

5. de Montpréville VT, Macchiarini P, Dulmet E. Thymic neuroendocrine carcinoma (carcinoid): a clinicopathologic study of fourteen cases. J Thorac Cardiovasc Surg 1996 Jan;111(1):134-141.

6. Fukai I, Masaoka A, Fujii Y, Yamakawa Y, Yokoyama T, Murase T, Eimoto T. Thymic neuroendocrine tumor (thymic carcinoid): a clinicopathologic study in 15 patients. Ann Thorac Surg 1999 Jan;67(1):208-211.

7. Soga J, Yakuwa Y, Osaka M. Evaluation of 342 cases of mediastinal/thymic carcinoids collected from literature: a comparative study between typical carcinoids and atypical varieties. Ann Thorac Cardiovasc Surg 1999 Oct;5(5):285-292.

8. Jansson JO, Svensson J, Bengtsson BA, Frohman LA, Ahlman H, Wängberg B, Nilsson O, Nilsson M. Acromegaly and Cushing's syndrome due to ectopic production of GHRH and ACTH by a thymic carcinoid tumor: in vitro responses to GHRH and GHRP-6. Clin Endocrinol (Oxf) 1998 Feb;48(2):243-250.

9. Okada S, Ohshima K, Mori M. The Cushing syndrome induced by atrial natriuretic peptide-producing thymic carcinoid. Ann Intern Med 1994 Jul;121(1):75-76.

10. Wick MR, Rosai J. Neuroendocrine neoplasms of the thymus. Pathol Res Pract 1988 Apr;183(2):188-199.

11. De Perrot M, Spiliopoulos A, Fischer S, Totsch M, Keshavjee S. Neuroendocrine carcinoma (carcinoid) of the thymus associated with Cushing's syndrome. Ann Thorac Surg 2002 Feb;73(2):675-681.

12. Gibril F, Chen YJ, Schrump DS, Vortmeyer A, Zhuang Z, Lubensky IA, Reynolds JC, Louie A, Entsuah LK, Huang K, et al. Prospective study of thymic carcinoids in patients with multiple endocrine neoplasia type 1. J Clin Endocrinol Metab 2003 Mar;88(3):1066-1081.

13. Filosso PL, Yao X, Ahmad U, Zhan Y, Huang J, Ruffini E, Travis W, Lucchi M, Rimner A, Antonicelli A, et al. Outcome of primary neuroendocrine tumors of the thymus: a joint analysis of the International Thymic Malignancy Interest Group and the European Society of Thoracic Surgeons databases. J Thorac Cardiovasc Surg 2015 Jan;149(1):103-109. 
14. Litvak A, Pietanza MC. Bronchial and thymic carcinoid tumors. Hematol Oncol Clin North Am 2016 Feb;30(1): 83-102.

15. Kayani I, Bomanji JB, Groves A, ,Conway G, Gacinovic S, Win T, Dickson J, Caplin M, Ell PJ. Functional imaging of neuroendocrine tumors with combined PET/CT using ${ }^{68} \mathrm{Ga}-$ DOTATATE (DOTA-DPhe1,Tyr3-octreotate) and 18F-FDG. Cancer 2008 Jun;112(11):2447-2455.

16. Binderup T, Knigge U, Loft A, Federspiel B, Kjaer A. 18F-fluorodeoxyglucose positron emission tomography predicts survival of patients with neuroendocrine tumors. Clin Cancer Res 2010 Feb;16(3):978-985.

17. Guidoccio F, Grosso M, Maccauro M, Orsini F, Perri M, Boni G, Banti E, Grassetto G, Rubello D, Mariani G, et al. Current role of 111In-DTPA-Octreotide scintigraphy in diagnosis of thymic masses. Tumori 2011 Mar-Apr;97(2): 191-195.

18. Granberg D, Sundin A, Janson ET, Oberg K, Skogseid B, Westlin JE. Octreoscan in patients with bronchial carcinoid tumors. Clin Endocrinol (Oxf) 2003 Dec;59(6):793-799.

19. Strosberg JR, Berry MF, Taxellar HD. Thymic neuroendocrine (carcinoid) tumors. UpToDate 2016 Oct.
20. Strosberg J, El-Haddad G, Wolin E, Hendifar A, Yao J, Chasen B, Mittra E, Kunz PL, Kulke MH, Jacene H, et al. Phase 3 trial of ${ }^{177} \mathrm{Lu}$-DOTATATE for midgut neuroendocrine tumors. N Engl J Med 2017 Jan;376(2):125-135.

21. Kayani I, Conry BG, Groves AM, Win T, Dickson J, Caplin M, Bomanji JB. A comparison of ${ }^{68} \mathrm{Ga}$-DOTATATE and ${ }^{18} \mathrm{~F}-\mathrm{FDG}$ $\mathrm{PET} / \mathrm{CT}$ in pulmonary neuroendocrine tumors. J Nucl Med 2009 Dec;1927-1932.

22. Reubi JC, Kvols LK, Waser B, ,Nagorney DM, Heitz PU, Charboneau JW, Reading CC, Moertel C. Detection of somatostatin receptors in surgical and percutaneous needle biopsy samples of carcinoids and islet cell carcinomas. Cancer Res 1990 Sep;50(18):5969-5977.

23. Lococo F, Perotti G, Cardillo G, De Waure C, Filice A, Graziano P, Rossi G, Sgarbi G, Stefanelli A, Giordano A, Granone P. Multicenter comparison of ${ }^{18} \mathrm{~F}$-FDG and ${ }^{68} \mathrm{Ga}$-DOTA-peptide PET/CT for pulmonary carcinoid. Clin Nucl Med 2015 Mar;40(3):e183-e189.

24. Isidori AM, Sbardella E, Zatelli MC, Boschetti M, Vitale G, Colao A, Pivonello R; ABC Study Group. Conventional and nuclear medicine imaging in ectopic Cushing's syndrome: a systematic review. J Clin Endocrinol Metab 2015 Sep;100(9):3231-3244. 